Available online at GSC Online Press Directory

GSC Biological and Pharmaceutical Sciences

e-ISSN: 2581-3250, CODEN (USA): GBPSC2

Journal homepage: https://www.gsconlinepress.com/journals/gscbps

(RESEARCH ARTICLE)

\title{
Iron deficiency in non-anemic individuals: a retrospective analysis of Libyan population
}

\author{
Elawamy Hayam Abdalla 1, ${ }^{*}$, Elmhdwi Maraia Farag ${ }^{2}$, Abdulla Sara Ali ${ }^{3}$, Jaber Nesrin Hussin ${ }^{1}$ and Algathafy \\ Khlid Gbril ${ }^{3}$
}

${ }^{1}$ Department of Medical Laboratory, College of Medical Technology, Benghazi, Libya.

2 Department of Chemistry, Faculty of Science, University of Benghazi, Benghazi, Libya.

${ }^{3}$ Department of Biochemistry, Faculty of Medicine, University of Benghazi, Benghazi, Libya.

Publication history: Received on 06 April 2020; revised on 17 April 2020; accepted on 18 April 2020

Article DOI: https://doi.org/10.30574/gscbps.2020.11.1.0088

\begin{abstract}
Iron deficiency may be severe despite a normal hemoglobin and full blood count. Since iron losses not met iron intake, iron stores depleted and non-anemic iron deficiency (NAID), occurs preceding iron deficiency anemia (IDA). NAID is a diagnostic challenge. Ferritin concentration is the most sensitive and specific test used for identification of iron deficiency. The study designed to determine the prevalence of Non-anemic iron deficiency (NAID). In addition, the correlation between serum ferritin with iron and hemoglobin levels in NAID. The study included (35 males and 110 females) non-anemic patients, recruited from the Al Akeed laboratory after completed a questionnaire to obtain clinical information and analyzed through SPSS version 24. Blood samples collected and analyzed for iron variables (Serum iron, total iron binding capacity (TIBC), transferrin saturation, ferritin, and hemoglobin (Hb). Based on ferritin levels non-anemic patients divided into two groups, the proportion of Normo-ferritin was $47.6 \%$ with normal iron level (96.7 \pm 50.8$), \mathrm{Hb}(13.5 \pm 2.0)$ UIBC $(250.4 \pm 103.3)$ and TIBC, (342.0 \pm 94.9$)$. Hemoglobin had significant correlation with $\mathrm{MCHC}, \mathrm{MCH}$, and MCV in this group. Low-ferritin level were $11.8 \pm 7.1 \mathrm{ng} / \mathrm{mL}$ in 76 cases suggesting the prevalence of sub-clinical iron deficiency in $52.4 \%$ cases. The levels of iron, Hemoglobin were normal. Ferritin had positive correlation with iron levels, Hemoglobin and MCH, and a highly significant but negative correlation with UIBC and TIBC. The ferritin level can be helpful in early recognition and diagnosis of IDA with appropriate interpretation of IDA. A direct relationship of iron, ferritin and transferrin with hemoglobin in non-anemic. Iron deficiency.
\end{abstract}

Keywords: Non-anemic iron deficiency (NAID); Ferritin; Transferrin; Anemia; Deficiency; Iron.

\section{Introduction}

Anemia is a serious global public health problem, which influences $43 \%$ of youngsters more youthful than five years old, $38 \%$ of pregnant women, and $29 \%$ of non-pregnant worldwide [1] Iron deficiency is prevalent etiology globally [2]. Iron deficiency anemia (IDA) can prompt can lead to developmental delays, behavioral disturbances, perinatal complications, and the failure of learning ability and cognitive function [3],[4], and early detection of IDA is essential in helping to prevent several complications and for improving patient nature of life [5].

Iron deficiency might be severe despite a normal hemoglobin and full blood count. Symptoms, which might delay, and debilitating, should raise clinical doubt on iron deficiency even if a full blood count is normal. A lifelong history of blood loss, such as abundant menstruation, pregnancies, blood donations, accidents/surgery as well as history of celiac disease, atrophic gastritis and medications restricting gastric acid secretion, must be taken [6]. Iron deficiency without anemia is a diagnostic challenge, as it may go unrecognized for drew out period moreover. There are a whole lot of

\footnotetext{
* Corresponding author: Elawamy Hayam Abdalla
} 
nothing-characterized indicative criteria. The doubt ought to emerge, if a patient with a typical full blood count presents indications of iron deficiency anemia [7],[8],[9] essentially together with low ferritin level and particularly when the clinical history upholding iron deficiency. Iron deficiency anemia (hemoglobin $\leq 130 \mathrm{~g} / \mathrm{L}$ in males and $\leq 120 \mathrm{~g} / \mathrm{L}$ in females) is a late manifestation of iron deficiency, both of which are common medical conditions in everyday clinical practice [8],[9],[10]. Some 10-20\% of menstruating women have iron deficiency, and 3-5\% of them are honestly anemic [10]. Iron deficiency (anemia) may regularly be asymptomatic and go undiscovered for a long time. The serum ferritin level (cut off $<30 \mu \mathrm{g} / \mathrm{L}$ ) is the most sensitive and specific test utilized for recognizable proof of iron inadequacy [8],[9]. However, in clinical laboratories, the lower limit of the reference range is often set at 10-20 $\mu \mathrm{g} / \mathrm{L}$ [11]. This may lead confusion, as the role of ferritin in the detection of iron deficiency is equivocal. Then the ferritin can be enhanced with evaluation of transferrin saturation, soluble transferrin receptor (sTfr) and the ratio between sTfr and logarithm of ferritin as well as hepcidin [8],[9]. The serum ferritin concentration (cut off $<30 \mu \mathrm{g} / \mathrm{L}$ ) is the most sensitive and specific test used [8],[9]. Serum ferritin level used to assess the level of iron storage and to diagnose iron deficiency state when the serum ferritin level is below $12 \mathrm{ng} / \mathrm{ml}$. This is the generally accepted cut-off level for serum ferritin by WHO, below which iron stores considered to deplete (WHO, 2001). This study was therefore, used serum ferritin level to determine the prevalence of Non-anemic iron deficiency (NAID) (sub-clinical iron deficiency), and to screen and evaluation of red blood cell (RBC) indices.

\section{Methods}

\subsection{Study design:}

Consecutive iron study samples from patients attending Al Akeed laboratory, Benghazi, Libya, Samples collected and prospectively analyzed at the hematology and biochemistry Laboratory.

\subsection{Exclusion criteria:}

Aged, smoking patients, alcoholic patient, nephrotic disease patient, malignancy, hepatitis, and patients suffering from renal failure or kidney disease excluded from the study.

\subsection{Study population:}

In this study, the respondents divided into two groups.

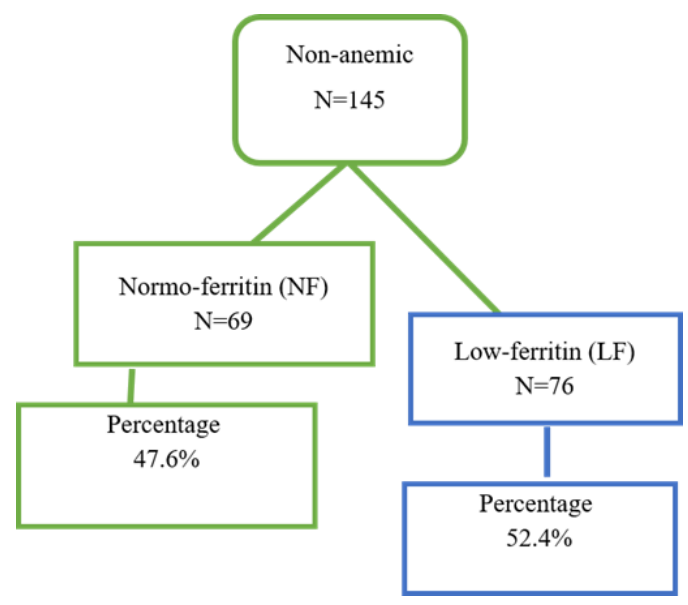

Figure 1 Study framework

\subsection{Blood sampling}

Blood venous samples drew from all the patients the blood collected in EDTA Plasma and plain tubes, and serum separated from plain tubes and stored at $20^{\circ} \mathrm{C}$ until the assays performed. The whole blood was analyzed for complete blood picture.

\subsection{Analytical methods}

Sera analyzed for iron, TIBC, UIBC, TIBC, ferritin and transferrin. Fully automated Cobas Integra 400 plus (ROCH, Germany) used for estimating iron profile. CBC machine DIAGON Company. 


\subsection{Statistical analysis}

The data analyzed using the statistical package for social sciences (SPSS version 24). Descriptive characteristics of the study participants calculated as mean standard deviation (SD). Patients divided in to two groups. Comparisons between groups were performed by analysis of variance (Correlation coefficient) were used to determine the differences in subject characteristics. Person correlation coefficient determination done to evaluate the degree of association between ferritin iron and hemoglobin parameter. P value (two- tailed) $<0.05$ was considered as statistically significant.

\section{Results}

\subsection{Characteristics of studying subjects}

On the basis of ferritin levels 145 non-anemic patients were divided into two groups, Group 1(NF), 47.6\% of patients with ferritin level $=95.4 \pm 61.1$ and Group $2(\mathrm{LF}), 52.4 \%$ of patients with low ferritin level $=11.8 \pm 7.1$. Age, Hb level, MCV, Iron, MCMC and MCH were lower in the Group 2 (LF), than the Group 1(NF), while the UIBC and TIBC were higher in the Group 2 (LF) look at table 1 Figure 2,3and 4.

Table 1 Show the iron and CBC variables in the Group 1(NF) and Group 2 (LF)

\begin{tabular}{lcc}
\hline Variables & $\begin{array}{c}\text { Normo-ferritin (NF) } \\
\mathbf{N = 6 9}\end{array}$ & $\begin{array}{c}\text { Low-ferritin (LF) } \\
\mathbf{N}=\mathbf{7 6}\end{array}$ \\
\hline Age & $40.0 \pm 14.9$ & $36.7 \pm 13$ \\
$\mathrm{Hb}$ & $13.5 \pm 2.0$ & $11.8 \pm 6.2$ \\
Ferritin & $95.4 \pm 61.1$ & $11.8 \pm 7.1$ \\
Iron & $96.7 \pm 50.8$ & $32.5 \pm 32.4$ \\
Ts\% & $29.8 \pm 17.8$ & $12.7 \pm 8.8$ \\
UIBC & $250.4 \pm 103.3$ & $367.3 \pm 74.7$ \\
TIBC & $342.0 \pm 94.9$ & $418.5 \pm 57.9$ \\
MCHC & $34.0 \pm 0.6$ & $32.0 \pm 5.4$ \\
MCH & $29.7 \pm 1.4$ & $26.3 \pm 3.2$ \\
MCV & $87.6 \pm 4.1$ & $79.7 \pm 7.3$ \\
\hline
\end{tabular}

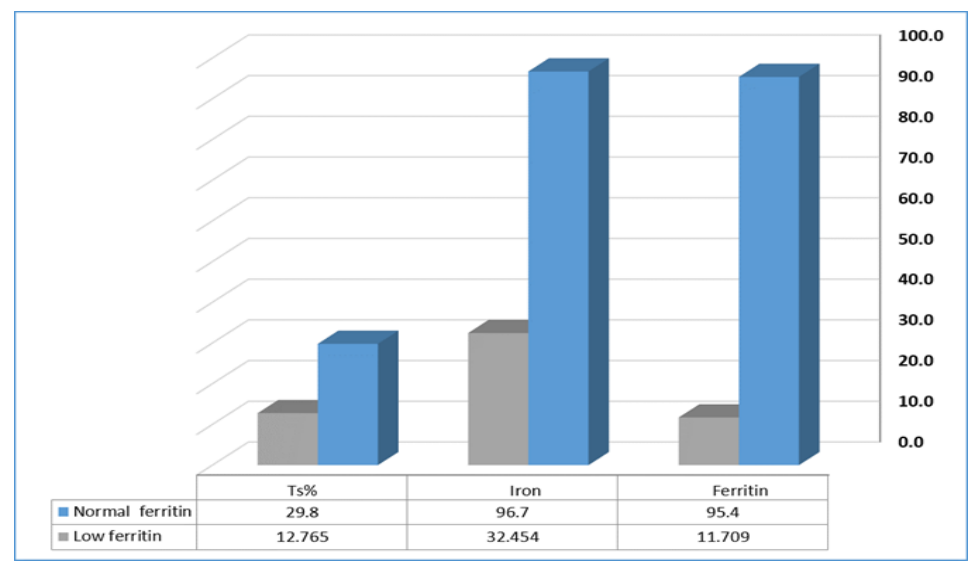

Figure 2 Show mean value, \pm standard division, of ferritin, iron and transferrin saturation percentage. 


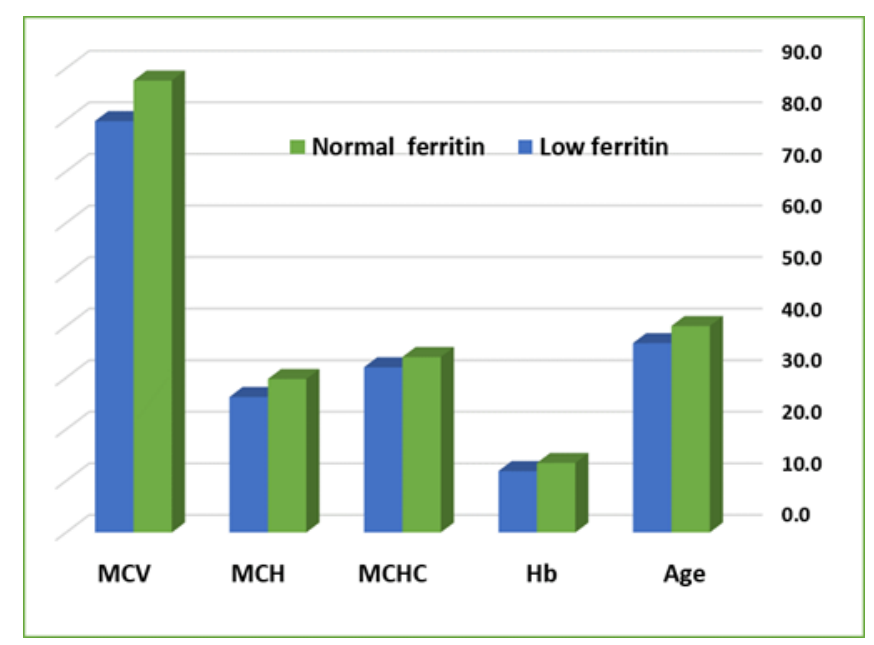

Figure 3 Show mean value of age, haemoglobin, MCHC, MCH, MCV.

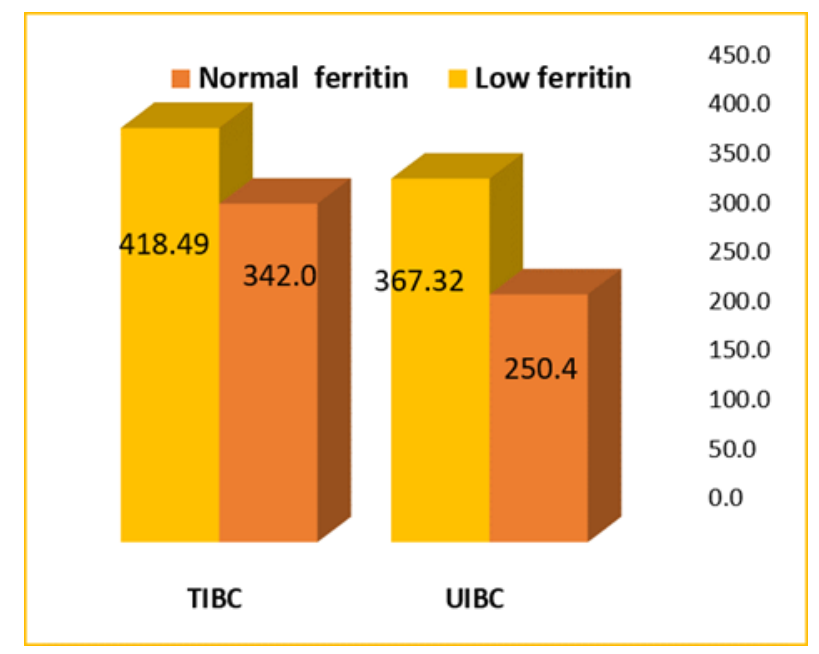

Figure 4 Show mean value of TIBC, UIBC.

\subsection{Factors contributing to NAID (male and female comparison):}

Table 2 presents the factors contributing to NAID. Factors associated with NAID in males were older age (39.7 \pm 14.7$)$ and higher in iron level $(98.2 \pm 42.9)$.

Ferritin level (108.8 \pm 78.5$)$, Ts (30.1 \pm 14.0$)$, Hb level (14.8 \pm 1.5$)$, MCHC (33.6 \pm 0.9$)$ and MCH (29.4 \pm 2.4$)$ MCV (87.5 \pm 5.5$)$, while in females they were younger age $(37.8 \pm 14.4)$, lower in iron level $(64.4 \pm 46.7)$, ferritin level $(33.5 \pm 37.2)$, Ts

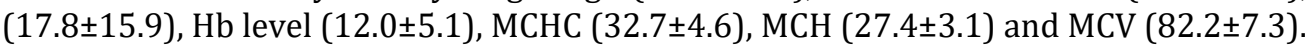

UIBC and TIBC lower in male $(240.5 \pm 75.2$ and $338.7 \pm 57.8$ respectively), than female $(335.9 \pm 105.4$ and $396.3 \pm 89.9$ respectively).look at table 2 , and figure 5 and 6. 
Table 2 Show mean values \pm standard division of age, iron, ferritin, HB, Ts\%, UIBC, TIBC, MCHC, MCH, MCV in male and female.

\begin{tabular}{lll}
\hline Variables & Male $\mathbf{N}=\mathbf{3 5}$ & Female $\mathbf{N}=110$ \\
\hline Age & $39.7 \pm 14.7$ & $37.8 \pm 14.4$ \\
Iron & $98.2 \pm 42.9$ & $64.4 \pm 46.7$ \\
Ferritin & $108.8 \pm 78.5$ & $33.5 \pm 37.2$ \\
Ts\% & $30.1 \pm 14.0$ & $17.8 \pm 15.9$ \\
UIBC & $240.5 \pm 75.2$ & $335.9 \pm 105.4$ \\
TIBC & $338.7 \pm 57.8$ & $396.3 \pm 89.9$ \\
Hb & $14.8 \pm 1.5$ & $12.0 \pm 5.1$ \\
MCHC & $33.6 \pm 0.9$ & $32.7 \pm 4.6$ \\
MCH & $29.4 \pm 2.4$ & $27.4 \pm 3.1$ \\
MCV & $87.5 \pm 5.5$ & $82.2 \pm 7.3$ \\
\hline
\end{tabular}

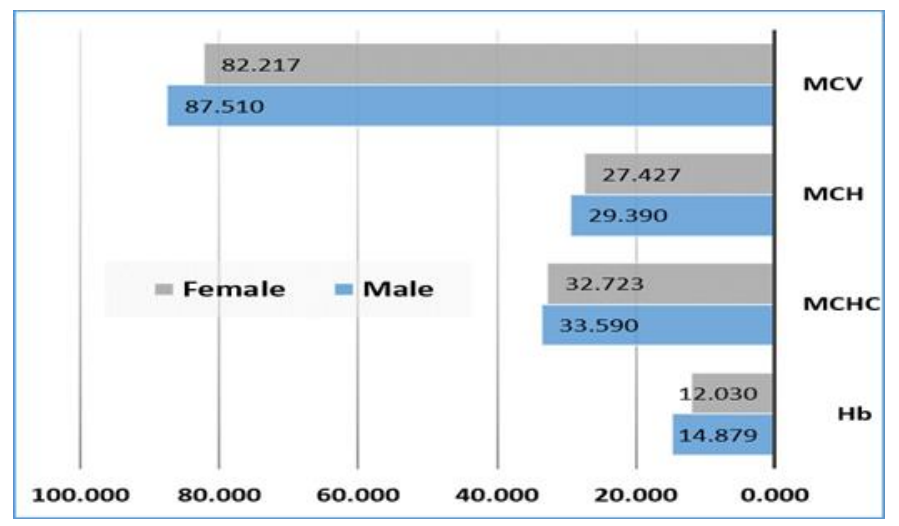

Figure 5 Show mean value of MCV, MCH, MCHC, haemoglobin.

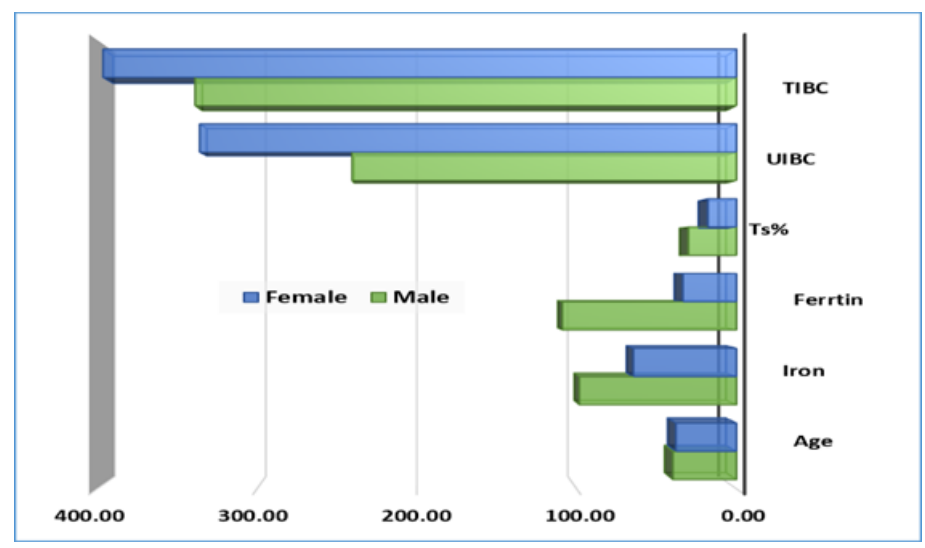

Figure 6 Show mean value of TIBC, UIBC, ferritin, iron, age.

\subsection{Iron correlation:}

Age, $\mathrm{Hb}$ and MCHC showed correlation with serum iron levels in NAID cases ( $\mathrm{r}-0.152,0.202$ and 0.313 respectively). While, it was not statistically significant except MCHC (Pv 0.069, 0.027, 0.017 respectively). However, Ts, ferritin, MCH 
and MCV showed a significant correlation with serum iron ( $\mathrm{r} 0.959,0.372,0.623$ and 0.595 respectively). UIBC and TIBC showed negative significant correlation with serum iron levels in NAID cases. ( $\mathrm{r}-0.645$ and -0.326 respectively) $(\mathrm{Pv}=0.000)$ look at table 3 .

Table 2 Show correlation coefficients (R) and (P-value) of ferritin with age, HB, Ts\% UIBC, TIBC, MCHC, MCH, MCV.

\begin{tabular}{cll}
\hline Variables & \multicolumn{2}{l}{ IRON } \\
\hline Age & -0.152 & 0.069 \\
HB & 0.202 & 0.027 \\
Ts\% & 0.959 & 0.000 \\
FERRTIN & 0.372 & 0.000 \\
UIBC & -0.645 & 0.000 \\
TIBC & -0.326 & 0.000 \\
MCHC & 0.313 & 0.017 \\
MCH & 0.623 & 0.000 \\
MCV & 0.595 & 0.000 \\
\hline
\end{tabular}

\subsection{Ferritin correlation:}

The age, $\mathrm{Hb}$ and MCHC showed correlation with serum ferritin levels in NAID cases (r 0.081, 0.208 and 0.168 respectively). However, it was not statistically significant expect hemoglobin ( $\mathrm{Pv}=0.023$ respectively).

Through Ts, Iron, MCH and MCV showed a significant correlation with serum ferritin (r 0.456, 0.372, 0.034 and 0.406, respectively).

The UIBC and TIBC showed negative significant correlation with serum ferritin levels in NAID cases ( $\mathrm{r}-0.519$ and 0.457 respectively) $(\mathrm{Pv}=0.000)$, look at table 4 .

Table 3 Show correlation coefficients (R) and (P-value) of ferritin with age, HB, Ts\%, UIBC, TIBC, MCHC, MCH, MCV.

\begin{tabular}{cll}
\hline Variables & \multicolumn{2}{c}{ Ferritin } \\
\hline Age & R & Pv \\
HB & 0.081 & 0.329 \\
Ts\% & 0.456 & 0.023 \\
Iron & 0.372 & 0.000 \\
UIBC & -0.519 & 0.000 \\
TIBC & -0.457 & 0.000 \\
MCHC & 0.168 & 0.2000 \\
MCH & 0.034 & 0.001 \\
MCV & 0.406 & 0.002 \\
\hline
\end{tabular}

\section{Discussion}

Iron deficiency (ID), with or without anemia, affects the retardation of verbal learning, memory, and cognitive function in children and adolescents [12],[13], and is also positively associated with global cognitive decline and the incidence 
of dementia in older people [14]. Additionally, ID decreases the work capacity and productivity of adults, finally resulting in a negative effect on national socioeconomic development [15].

The development of ID involves three sequentially affects: iron depletion, iron-deficient erythropoiesis, and ID anemia [16]. A negative iron balance first decreases the body iron reserves. Iron depletion results from an absence of iron in the storage compartment. Once iron stores depleted, the amount of iron in the transport compartments starts to decrease. The stage of iron-deficient erythropoiesis reached, when the iron supply to the erythropoietic marrow is insufficient for erythropoiesis, but with the hemoglobin ( $\mathrm{Hb}$ ) levels still being normal. This condition called non-anemic iron deficiency anemia when the functional compartment is finally affected and the iron supply is no longer sufficient to produce a normal $\mathrm{Hb}$ level, ID anemia becomes apparent.

Consequently, ID can remain undetected by screening using cut-offs of the complete blood count (CBC) including the Hb level in health check-ups.

The present study is the first national study of anemia incorporating both sexes in Libya. The prevalence rate of anemia was 52.6\%. Our study showed that females they were lower in iron level, ferritin level. Ts, RBC parameter, and the results of this study are consistent with most studies in the literature. Le et al., which suggested that women of reproductive age had a high prevalence of anemia [17], while a study conducted in Saudi Arabia indicated that the prevalence of anemia in adolescent girls was higher than that in adolescent males [18]. Ismail et al, stated that the prevalence of anemia in females was almost double that in males in a study conducted in Kerala, India [19].

Prolonged insufficient iron supply to tissues eventually leads to IDA, which characterized by low transferrin saturation (Ts), ferritin, MCH and MCV [20],[21]. This is going with our finding since serum iron had significant correlations with Ts, Ferritin, MCH and MCV, and negative significant correlation with the UIBC and TIBC levels in NAID cases.

As for correlation of ferritin levels with red cell indices in all the 145 cases, no significant correlation were found between ferritin and the age, Hb and MCHC. In the whole group Ts, Iron, MCH and MCV showed significant, positive correlation with serum ferritin these findings were similar to Archer study[22].

UIBC and TIBC were had negative strong correlation and highly significant. Hemoglobin had significant correlation and $\mathrm{MCHC}, \mathrm{MCH}, \mathrm{MCV}$.

This finding is in line with data in previous studies in iron deficiency Measurement of both serum ferritin and Ts offers a straightforward means to identify the presence of iron deficiency in these at-risk groups [23].

The limitations were faced in the study were. First, the study design did not include socioeconomic and nutritional factors that could also be risk factors for ID. Second, other markers for ID such as soluble transferrin saturation receptor, hepcidin or zinc protoporphyrin could not use to diagnose SID in this study. However, those markers also have limitations and the cost of testing needs to consider. Finally, small sample size and further studies with larger sample size may help confirming these findings.

\section{Conclusion}

This study found that the overall prevalence of subclinical iron deficiency in non-anemic (SID). Iron deficiency regularly stays undiscovered and untreated in the typical iron and hemoglobin levels patients. It may not suspected in light of the fact that the average side effects. Estimation of both serum ferritin and Ts offers a clear way to recognize the nearness of iron inadequacy in these in danger gatherings. Searching for the pathologic causes of ID could be helpful for health promotion and preventing subsequent diseases.

\section{Compliance with ethical standards}

\section{Acknowledgments:}

The authors would like to thank Al Akeed Company and the worker in it for their cooperative with us and supplied us by cases results and all clinical information about them.

\section{Disclosure of conflict of interest}

No observed conflict of interest among the authors. 


\section{Statement of ethical approval}

Informed consent obtained from all participants, and the Ethics Review Board of the University of Benghazi approved the study design.

\section{Statement of informed consent:}

Informed consent was signed to all respondents before the data collection.

\section{References}

[1] Pasricha R and Drakesmith H. (2016). "Iron Deficiency Anemia: Problems in Diagnosis and Prevention at the Population Level," Hematol. Oncol. Clin. North Am., 30(2), 309-325.

[2] Martin H, Kimanya M and Mosha T. (2018). "Prevalence and predictors of anemia among children under 5 years of age in Arusha," Pediatr. Heal. Med. Ther., 9-15.

[3] Kiudeliene R, Griniute R and Labanauskas L. (2008). "Prognostic value of reticulocyte hemoglobin content to diagnose iron deficiency in 6-24-month-old children," Medicina (B. Aires), 44(9), 673-677.

[4] Hempel E and Bollard E. (2016). “The Evidence-Based Evaluation of Iron Deficiency Anemia," Med. Clin. North Am., 100(5), 1065-1075.

[5] Jimenez K, Kulnigg-Dabs S and Gasche C. (2015). "Management of iron deficiency Anemia," Gastroenterol. Hepatol., 11(4), 241-250.

[6] Soppi E. (2018). "Iron deficiency without anemia - a clinical challenge," Clin. Case Reports, 6(6), 1082-1086.

[7] Sawada T, Konomi A and Yokoi K. (2014). "Iron deficiency without anemia is associated with anger and fatigue in young Japanese women," Biol. Trace Elem. Res., 159(1-3), 22-31.

[8] Camaschella C. (2015). Iron-deficiency anemia. N. Engl. J. Med., 372, 1832-1843.

[9] Lopez A, Cacoub P, Macdougall I and Peyrin-Biroulet L. (2016). "Iron deficiency anaemia," Lancet, 387(10021), 907-916.

[10] Umbreit J. (2005). "Iron deficiency: A concise review," Am. J. Hematol., 78(3), 225-231.

[11] Goodnough L, Nemeth E and Ganz T. (2010). "Detection, evaluation, and management of iron-restricted erythropoiesis." Blood, 116(23), 4754-4761.

[12] Halterman J, Kaczorowski J, Aligne C, Auinger P and Szilagyi P. (2001). “Iron deficiency and cognitive achievement among school-aged children and adolescents in the United States.," Pediatrics, 107(6), 1381-1386.

[13] WHO. (2001). "Iron deficiency anaemia".

[14] Andro M, Le Squere P, Estivin S and Gentric A. (2013). "Anaemia and cognitive performances in the elderly: a systematic review" Eur. J. Neurol., 20(9), 1234-1240.

[15] Baltussen R, Knai C and Sharan M. (2004). "Iron Fortification and Iron Supplementation are Cost-Effective Interventions to Reduce Iron Deficiency in Four Subregions of the World," J. Nutr., 134(10), 2678-2684.

[16] Skikne B, Lynch S, Borek D and Cook J. (1984). "Iron and blood donation" Clin. Haematol., 13(1), 271-287.

[17] Le C. (2016). "The Prevalence of Anemia and Moderate-Severe Anemia in the US Population (NHANES20032012).," PLoS One, 11(11), e0166635.

[18] Alquaiz A. et al., (2013). "Prevalence of anemia and associated factors in child bearing age women in Riyadh, Saudi Arabia," J. Nutr. Metab., 10-16.

[19] Ismail I, Kahkashan A, Antony A and VKS. (2016). "Role of socio-demographic and cultural factors on anemia in a tribal population of North Kerala, India," Int. J. Community Med. Public Heal., 1183-1188.

[20] Toxqui L and Vaquero M. (2015). "Chronic Iron Deficiency as an Emerging Risk Factor for Osteoporosis: A Hypothesis," 2324-2344.

[21] Blanco-Rojo R, Pérez-Granados A, Toxqui L, González-Vizcayno C, Delgado M and Vaquero M. (2011). “Efficacy of a microencapsulated iron pyrophosphate-fortified fruit juice: A randomised, double-blind, placebo-controlled study in Spanish iron-deficient women," Br. J. Nutr., 105(11), 1652-1659. 
[22] Cappellini M, De Francisco J, Dignass A, Doehner W, Lam C and Macdougall I. (2017). "Iron deficiency across chronic inflammatory conditions : International expert opinion on definition, diagnosis and management," 10681078.

[23] Archer N and Brugnara C and “Diagnosis of iron-deficient states”. (2015). Crit. Rev. Clin. Lab. Sci., 1-17.

\section{How to cite this article}

Elawamy HA, Elmhdwi MF, Abdulla SA, Jaber NH and Algathafy KG. (2020). Iron deficiency in non-anemic individuals: a retrospective analysis of Libyan population. GSC Biological and Pharmaceutical Sciences, 11(1), 171-179. 